\title{
Síndroma de roubo da subclávia: um caso clínico de aparente hipotensão
}

Diana Patrícia Pereira Coelho, ${ }^{1}$ Inês José Maia da Silva, ${ }^{1}$ Hiroshi Okai ${ }^{1}$

\section{RESUMO}

Introdução: A estenose da artéria subclávia proximal, condicionando a síndroma de roubo da subclávia, é frequentemente secundária a doença arterial aterosclerótica. Pode ser totalmente assintomática ou cursar com sintomas de isquemia do membro superior ipsilateral ou sintomas de isquemia transitória da circulação cerebral posterior. Apesar de o diagnóstico definitivo ser imagiológico, as alterações ao exame objetivo são fortemente sugestivas, nomeadamente um diferencial de pressão arterial entre os dois membros superiores.

Descrição do caso: Mulher de 69 anos, com HTA, DM tipo 2, dislipidemia e obesidade, recorreu à consulta por tonturas, cefaleias e descida dos valores tensionais, com dois meses de evolução, tendo decidido suspender a medicação anti-hipertensora. Referia episódio prévio de cefaleia, disartria súbita e sensação de "língua presa", que motivou ida ao serviço de urgência. Neste, quando observada, já não apresentava qualquer alteração objetiva. No exame físico, na consulta do MF, destacava-se uma PA 108/75 mmHg, avaliada no membro superior esquerdo. Devido aos vários fatores de risco ateroscleróticos e ao episódio prévio sugestivo de AIT foram pedidos exames complementares de diagnóstico, incluindo ecodoppler dos vasos cervicais, que demonstrou um fluxo retrógrado da artéria vertebral esquerda, sugestivo de estenose da artéria subclávia esquerda proximal. Na sequência, um EO minucioso revelou um diferencial de PA, entre os dois membros superiores (108/79mmHg à esquerda e 170/81 à direita). Foi recomendado retomar a medicação anti-hipertensora, reforçado o controlo dos múltiplos fatores de risco ateroscleróticos e referenciada a cirurgia vascular.

Comentário: Neste caso, os valores tensionais eram aparentemente diminuídos; contudo, após a avaliação da pressão arterial em ambos os membros superiores, constatou-se que a utente mantinha uma HTA grau 2 não controlada. Os autores pretendem alertar para a importância de, no meio das muitas tarefas do dia-a-dia, ser mantida uma boa prática semiológica na determinação da pressão arterial, em ambos os braços.

Palavras-chave: Hipotensão; Determinação da pressão arterial; Síndroma de roubo da subclávia; Aterosclerose.

\section{INTRODUÇÃO}

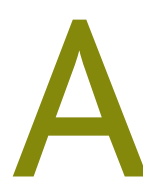
síndroma de roubo da subclávia descreve um fluxo sanguíneo retrógrado na artéria vertebral associada à estenose severa ou oclusão da artéria subclávia proximal ipsilateral. ${ }^{1}$ Distalmente à obstrução ocorre uma redução da pressão na artéria subclávia, causando um fluxo retrógrado de sangue na artéria vertebral ipsilateral à estenose, que deveria ser distribuído pelas artérias basilar, vertebral contralateral e carótida, "roubando", assim, sangue da circulação cerebral. É uma entidade relativamente rara, estando relatada na literatura uma prevalência entre

1. USF Vale do Vouga, ACeS Aveiro Norte. São João da Madeira, Portugal.
$0,6 \%$ a $6,4 \%,{ }^{2}$ sendo mais comum nos homens acima dos 50 anos. $^{2-3}$

A doença arterial aterosclerótica é a principal causa de doença oclusiva da artéria subclávia e afeta mais comummente a artéria subclávia esquerda. ${ }^{1}$ Outras causas menos comuns incluem arteriopatias inflamatórias (e.g., arterite de Takayasu ou arterite de células gigantes), anomalias congénitas, estados pós-cirúrgicos ou a síndroma de compressão do desfiladeiro torácico. ${ }^{1}$

Os doentes com fluxo retrógrado da artéria vertebral são maioritariamente assintomáticos; raramente podem apresentar sintomas de isquemia dos membros superiores devido à redução do fluxo arterial (como cãibras musculares secundárias depois de um trabalho 
vigoroso com os braços elevados acima da cabeça) ou sintomas neurológicos consistentes com isquemia transitória da circulação cerebral posterior (como tontura, vertigem, síncope, disartria, alterações visuais secundárias à disfunção vestibular, nistagmo ou diplopia). ${ }^{1}$ Apesar de tudo, estes episódios de isquemia cerebral transitória raramente progridem para acidente vascular cerebral ${ }^{1-3}$ e raramente resultam em défice neurológico permanente. ${ }^{4}$

O diagnóstico é frequentemente feito por ecodoppler das artérias carótidas e artéria subclávia. ${ }^{1,5}$ Contudo, as alterações no exame objetivo podem induzir um elevado nível de suspeição clínica. O principal sinal é um diferencial de pressão arterial (PA), entre os dois membros superiores, superior ou igual a $20 \mathrm{mmHg} .^{1-3}$ Outros sinais são um pulso radial ou cubital diminuído ou ausente à palpação, a auscultação de um sopro na subclávia e um teste de roubo da subclávia positivo (hiperemia reativa no braço, provocada pela manobra do cuff durante a medição da PA). ${ }^{3}$

O presente caso clínico relata uma aparente descida dos valores tensionais que se mostrou ser uma estenose da artéria subclávia proximal. A história clínica detalhada e o exame físico pormenorizado, incluindo a determinação da PA em ambos os membros superiores, são suficientes para a sua suspeita clínica. Assim, os autores pretendem alertar para eventuais lapsos de diagnóstico decorrentes da avaliação sistemática da PA num único braço, lembrando que está recomendada a determinação da PA em ambos os braços, pelo menos na primeira consulta ou sempre que ocorram variações significativas da PA em doentes hipertensos.

\section{DESCRIÇÃO DO CASO}

O caso clínico reporta-se a uma mulher de 69 anos, de raça caucasiana. Concluiu o ensino básico e atualmente encontra-se reformada da profissão de operária fabril. No que respeita à caracterização familiar é casada e pertencente a uma família nuclear na fase VIII do ciclo de Duvall.

Dos antecedentes pessoais destacam-se hipertensão arterial (HTA) sem complicações, desde 2008, diabetes mellitus (DM) tipo 2 diagnosticada em 2014, dislipidemia, obesidade, hipotiroidismo e neoplasia maligna do cego diagnosticada em 2017, tendo realizado hemicolectomia direita laparoscópica. Medicada habi- tualmente com ramipril 2,5mg qd, metformina $850 \mathrm{mg}$ bid, atorvastatina $20 \mathrm{mg}$ qd, gemfibrozil $600 \mathrm{mg} q d$, levotiroxina sódica $0,112 \mathrm{mg}$ qd e clopidogrel $75 \mathrm{mg} \mathrm{qd}$. Sedentária, sem hábitos tabágicos ou alcoólicos. Plano nacional de vacinação atualizado. Sem antecedentes familiares de relevo.

A utente é seguida regularmente na consulta de vigilância de HTA e DM pelo seu médico de família. Na consulta, do dia 17 de julho de 2020, a utente refere tonturas e cefaleias diárias, com dois meses de evolução e que os seus valores médios de pressão arterial (PA), em ambulatório, eram de 100/65mmHg, pelo que suspendeu por autoiniciativa a toma de ramipril $2,5 \mathrm{mg}$. Referiu ainda que, no dia 18 de maio de 2020 , recorreu ao serviço de urgência (SU) com queixas de cefaleia intensa na região parietal direita, sensação da "língua presa" e disartria de início súbito. No SU, quando observada, já não apresentava qualquer alteração ao exame objetivo, incluindo o exame neurológico, tendo sido realizada apenas pesquisa de SARS-CoV-2 por PCR, com resultado negativo.

Ao exame objetivo apresentava peso $93,5 \mathrm{~kg}$, altura $1,63 \mathrm{~m}$, IMC $35,19 \mathrm{~kg} / \mathrm{m}^{2}$ (obesidade grau II), perímetro abdominal $108 \mathrm{~cm}$; apirética, eupneica em ar ambiente, frequência cardíaca 70bpm e PA 108/75mmHg (avaliada no membro superior esquerdo). Mucosas coradas e hidratadas. Auscultação cardíaca com S1 e S2 audíveis em todos os focos auscultatórios, regulares, sem sopros audíveis. Auscultação pulmonar com murmúrio vesicular presente e simétrico bilateralmente, crepitações na base direita. Membros inferiores quentes de coloração normal, sem edemas periféricos. No exame neurológico sumário, a utente encontrava-se orientada no tempo e no espaço, com discurso coerente e fluente, sem disartria. Campos visuais normais. Pupilas simétricas e isocóricas, olhos na linha média em repouso. Face sem assimetrias em repouso, com mímica facial preservada e simétrica. Sem desvio à protusão da língua. Sem dismetria na prova dedo-nariz. Membros sem atrofias, sem alterações na força nem sensibilidade. Sem alterações na marcha.

Do estudo analítico, realizado a 29 de janeiro de 2020, destacava-se hemograma completo, função renal, hepática e tiroidea sem alterações, HbAlc 6,8\% e perfil lipídico com LDL 123,8mg/dl. O eletrocardiograma (ECG) em repouso, realizado a 1 de junho de 2020, apresentava um ritmo sinusal, com frequência cardíaca de 
68bpm e ausência de critérios de hipertrofia ventricular esquerda.

Face à coexistência de múltiplos fatores de risco para doença aterosclerótica e ao episódio sugestivo de acidente isquémico transitório (AIT) foram pedidos os seguintes exames complementares de diagnóstico: ecocardiograma transtorácico (ecoTT), ecodoppler dos vasos cervicais e tomografia computorizada cerebral (TC-CE). Relativamente à descida dos valores tensionais optou-se por manter a suspensão do ramipril $2,5 \mathrm{mg}$, para descartar eventual iatrogenia induzida pela medicação anti-hipertensora, e pedir registos de automedições de PA, em ambulatório, para a próxima consulta (agendada para o mês seguinte).

Após um mês, a utente voltou à consulta com valores médios de PA em ambulatório, avaliada no braço esquerdo, de 105-91/71-55mmHg e com os resultados dos exames prescritos. No ecoTT observavam-se sinais de remodeling concêntrico, insuficiência mitral mínima e função sistólica do ventrículo esquerdo preservada (FEVE 69\%). O ecodoppler dos vasos cervicais, à direita, mostrava "mínimas placas de ateroma calcificadas ao nível da carótida comum, bolbo carotídeo e artérias carótidas interna e externa" e, à esquerda, revelava "mínimas placas de ateroma calcificadas ao nível da artéria carótida comum, bolbo carotídeo e artérias carótida interna e externa, a artéria vertebral apresenta fluxo retrógrado, sugerindo estenose da artéria subclávia proximal". Na TC-CE eram visíveis "calcificações vasculares dos sifões carotídeos".

Perante a suspeita de fenómeno de roubo da subclávia, nesta segunda consulta foi avaliada a PA em ambos os membros superiores (MS), registando-se um valor de 108/79mmHg no MS esquerdo e $170 / 81 \mathrm{mmHg}$ no MS direito. Foi também avaliada a PA em ambos os membros inferiores (MI), tendo-se registado 169/90 mmHg no MI esquerdo e 169/86mmHg no MI direito. O restante exame objetivo não mostrava alterações, nomeadamente a auscultação cardiopulmonar normal, a auscultação carotídea sem sopros bilateralmente e os membros inferiores de coloração normal, com pulsos radiais amplos e simétricos, sem edemas periféricos.

Foi explicado à utente o diferencial de valores tensionais entre os dois MS. A aparente diminuição dos valores tensionais no MS esquerdo correspondia a uma diminuição do fluxo sanguíneo por estenose da artéria subclávia proximal esquerda. Nas restantes medições de PA (MS direito e MI) mantinha valores tensionais elevados, que seriam semelhantes aos seus valores tensionais sistémicos.

Assim, perante uma utente com HTA não controlada, com múltiplos fatores de risco ateroscleróticos e doença arterial periférica recomendou-se retomar a medicação anti-hipertensora. Foi sugerida a automedição da PA em ambulatório, no MS direito, para posterior ajuste gradual da medicação anti-hipertensiva até otimização dos valores tensionais. Nos doentes hipertensos diabéticos, o objetivo será a redução da TA para valores < 130/80mm Hg. ${ }^{6}$ Perante um risco cardiovascular muito alto optou-se por controlar a dislipidemia com atorvastatina $20 \mathrm{mg}$ qd e ezetimibe $10 \mathrm{mg}$ qd para valores de colesterol LDL alvo inferiores a 55 $\mathrm{mg} / \mathrm{dL}$. Manteve-se a antiagregação plaquetária simples com clopidogrel 75mg e a medicação antidiabética oral. A utente foi aconselhada a implementar, simultaneamente, modificações ao seu estilo de vida, nomeadamente a reduzir o peso, a adotar uma dieta rica em vegetais, com baixo teor de gorduras e sal e a praticar atividade física regular. Face à necessidade de esclarecimento etiológico e eventual intervenção terapêutica foi referenciada à consulta de cirurgia vascular.

\section{COMENTÁRIO}

Neste caso clínico, face à descida mantida dos valores tensionais e ao episódio sugestivo de AIT optou-se por suspender a medicação anti-hipertensora e efetuar um estudo dos territórios vasculares. O ecodoppler dos vasos cervicais mostrou um fluxo retrógrado na artéria vertebral esquerda sugestivo de estenose da artéria subclávia esquerda proximal. Perante este achado realizou-se um exame objetivo mais pormenorizado, incluindo a determinação da PA nos quatro membros, tendo-se constatado que a aparente diminuição dos valores tensionais correspondia a uma diminuição do fluxo sanguíneo no membro superior esquerdo, enquanto nos restantes membros apresentava valores tensionais compatíveis com HTA grau 2 não controlada.

Assim, recomenda-se que a PA no consultório seja medida em ambos os braços, pelo menos na primeira consulta, pois uma diferença da PAS > 15mmHg é sugestiva de doença ateromatosa e está associada a risco cardiovascular acrescido. ${ }^{7}$ No caso de uma diferença 
significativa da PAS (> 10mmHg) e consistente entre os dois braços, o que acarreta um risco cardiovascular aumentado, recomenda-se que as leituras posteriores sejam feitas no braço com valor mais elevado. ${ }^{7-8}$ Devem ser registados os valores da PA, bem como o braço em que foi medida, o tamanho da braçadeira utilizada, a hora da medição e alguma circunstância particular, como stress, febre ou agitação. ${ }^{9}$

Nesta utente, o fluxo retrógrado na artéria vertebral esquerda justificava também os sintomas neurológicos sugestivos de isquemia transitória da circulação cerebral posterior (tonturas e disartria transitória), devido à síndroma de roubo da artéria subclávia. A TC-CE desta utente não revela áreas de isquemia permanente, o que está de acordo com o que a literatura descreve para a maioria dos doentes. Uma vez que esta patologia coexiste frequentemente com outras doenças ateroscleróticas e pode mascarar outros distúrbios vasculares deve ser considerada nos diagnósticos diferenciais de doentes com sintomas como AIT ou angina cardíaca. ${ }^{5}$

A doença aterosclerótica é uma doença multissistémica, mas atinge eletivamente determinados territórios vasculares, onde o fluxo é mais turbulento, como a aorta torácica descendente, a aorta abdominal, artérias coronárias, as artérias poplíteas, as carótidas internas e o polígono de Wills. ${ }^{10}$ Assim, nesta utente, perante a suspeita de síndroma de roubo da artéria subclávia e tendo em conta a coexistência de múltiplos fatores de risco para doença aterosclerótica, era importante prevenir e tratar, agressivamente, os fatores de risco ateroscleróticos e prosseguir com o estudo de outros territórios vasculares, nomeadamente a aorta abdominal e artérias poplíteas. Contudo, este estudo não foi realizado nos cuidados de saúde primários, na medida em que a utente foi referenciada a consulta hospitalar, por haver uma possível correlação entre os sintomas neurológicos e a síndroma de roubo da subclávia.

O presente caso clínico relata uma aparente descida dos valores tensionais devido à medição sistemática da PA no MS esquerdo (braço não dominante) e que se revelou ser devida a uma estenose da artéria subclávia proximal. Neste caso, a suspensão da medicação anti-hipertensiva pela aparente hipotensão arterial pode ter contribuído para a posterior crise hipertensiva e o AIT. Assim, os autores pretendem alertar para a importância de, no meio das muitas tarefas do dia-a-dia, ser mantida boa prática semiológica na avaliação da PA em ambos os MS, pelo menos na primeira consulta ou sempre que ocorram variações significativas da PA em doentes hipertensos com provável doença aterosclerótica, identificando o membro que apresenta valores mais elevados, de modo a melhor caracterizar o doente e decidir o plano terapêutico mais adequado para o controlo dos fatores de risco modificáveis e a redução dos eventos vasculares agudos.

\section{REFERÊNCIAS BIBLIOGRÁFICAS}

1. Bayat I, Chuen J. Subclavian steal syndrome. Medscape [Internet]; 2019 [updated 2021 Mar 8; cited 2020 Oct 4]. Available from: https://emedicine.medscape.com/article/462036-overview

2. Tan TY, Schminke U, Lien LM, Tegeler $\mathrm{CH}$. Subclavian steal syndrome: can the blood pressure difference between arms predict the severity of steal? J Neuroimaging. 2002;12(2):131-5.

3. Granito S, Azevedo I, Jardim M, Andrade JL, Lélis M, Chaves A, et al. Aterosclerose: a propósito do fenómeno de roubo da subclávia [Atherosclerosis: apropos of a subclavian steal phenomenon]. Rev Soc Port Med Interna. 2011;18(1):35-9. Portuguese

4. EhrenfeldWK, Chapman RD,Wylie EJ. Management of occlusive lesions of the branches of the aortic arch. Am J Surg. 1969;118(2):263-43.

5. Nicholls SC, Koutlas TC, Strandness DE. Clinical significance of retrograde flow in the vertebral artery. Ann Vasc Surg. 1991;5(4):331-6.

6. Direção-Geral da Saúde. Diagnóstico, tratamento e controlo da hipertensão arterial: circular normativa n. ${ }^{\circ}$ 2/DGCG, de 31/03/2004. Lisboa: DGS; 2004.

7. European Society of Cardiology. Hipertensão: recomendações da ESC/ESH para o tratamento da hipertensão arterial: recomendações de bolso de 2018 da ESC [Internet]. Lisboa: Sociedade Portuguesa de Cardiologia; 2018. Available from: https://spc.pt/wp-content/uploads/ 2019/10/Pocket-guidelines-Hipertens\%C3\%A3o.pdf

8. European Society of Cardiology. Tradução Portuguesa das guidelines de 2013 da ESH/ESC para o tratamento da hipertensão arterial. Rev Port Hipertens Risco Cardiovasc. 2014;(39 Suppl).

9. Direção-Geral da Saúde. Hipertensão arterial: definição e classificação: norma n. ${ }^{\circ}$ 20/2011, de 28/09/2011, atualizada em 19/03/2013. Lisboa: DGS; 2013.

10. Kumar V, Cotran RS, Robins SL. Atherosclerosis. In: Kumar V, Cotran RS, Robins SL, editors. Basic pathology. 6th ed. Saunders; 1997. p. 282-9.

\section{CONFLITO DE INTERESSES}

Os autores declaram não possuir quaisquer conflitos de interesse.

\section{ENDEREÇO PARA CORRESPONDÊNCIA}

Diana Patrícia Pereira Coelho

E-mail: dianappcoelho@gmail.com

https://orcid.org/0000-0003-2383-4481

Recebido em 24-10-2020

Aceite para publicação em 25-05-2021 


\section{ABSTRACT}

\section{SUBCLAVIAN STEAL SYNDROME: APPARENTLY A HYPOTENSION CLINICAL CASE}

Introduction: The proximal subclavian stenosis associated with subclavian steal syndrome is frequently secondary to atherosclerotic arterial disease. It can be completely asymptomatic or present symptoms of superior ipsilateral arm ischemia or transitory posterior-circulation cerebral ischemia. The final diagnosis is imagiological. However, the alterations to the physical examination (PhE) are extremely suggestive, namely, arterial pressure discrepancy between the two superior arms.

Case description: A 69-year-old woman, with hypertension, diabetes mellitus type 2, dyslipidaemia, and obesity, has gone to the medical appointment with dizziness, headache and a decrease in blood pressure which had two months of evolution. She decided to suspend the antihypertensive treatment by herself. She also referred to a previous headache episode, sudden dysarthria, and a 'tied tongue' sensation, which let her go to emergency care. In urgency care, when she was observed, no objective alterations were found. During the family physician appointment, PhE showed a blood pressure $108 / 75 \mathrm{mmHg}$, which was evaluated on the superior left arm. Due to the various atherosclerosis risk factors and the previous episode suggestive of AIT, complementary diagnostic exams were made, including carotid and vertebral doppler ultrasonography, which showed a retrograde flow from the left vertebral artery that suggests proximal left subclavian stenosis. Consequently, a detailed PhE revealed a blood pressure discrepancy between the two superior arms $(108 / 79 \mathrm{mmHg}$ on the left and $170 / 81 \mathrm{mmHg}$ on the right). The woman was recommended to retake antihypertensive medicines, to control the multiple atherosclerosis risk factors and she was tracked to a vascular surgery appointment.

Comments: In this clinical case the BP values were, apparently, decreased. However, after an evaluation in both superior arms, it was revealed that the patient presented uncontrolled grade 2 hypertension. The authors really want to alert for the importance of keeping the good semiological practice of BP determination, in both arms, even though the multitasking daily routines.

Keywords: Hypotension; Blood pressure determination; Subclavian steal syndrome; Atherosclerosis. 